\title{
Analizando la tensión laboral de los psicólogos del ámbito clínico, mediante su resiliencia y bienestar
}

\author{
Analyzing the work stress of psychologists in the clinical field, through \\ their resilience and well-being
}

Ofelia Calero Escurra ${ }^{1}$

Ministerio del Trabajo y Promoción del Empleo

Recibido: $17-09-20$

Aceptado: $16-11-20$

Publicado: $21-12-20$

\section{Resumen}

La finalidad del presente trabajo de investigación fue establecer si existe asociación entre la resiliencia y el bienestar psicológico en psicólogos que laboran en el ámbito clínico, para ello se seleccionó a 93 psicólogos clínicos alumnos de posgrado en una universidad pública de la ciudad de Lima, los mismos que fueron evaluados con el test de Sánchez Cánovas de Bienestar psicológico (EBP) y el test de Wagnild y Young de Resiliencia (ER). Los resultados generales muestran una correlación positiva moderada, estadísticamente significativa entre la resiliencia y el bienestar psicológico, es decir, a medida que, en los psicólogos que laboran en el ámbito clínico, con formación de posgrado, aumenta la capacidad de poder transformar con éxito las adversidades que les surjan en la vida, permitiéndoles adaptarse y proyectarse exitosamente a su contexto también se acrecienta su percepción acerca de su satisfacción personal.

Palabras clave: Resiliencia; Bienestar; Psicólogos clínicos; Bienestar en las relaciones de pareja y Ecuanimidad.

\begin{abstract}
The purpose of this research work was to establish whether there is an association between resilience and psychological well-being in psychologists working in the clinical field, for this, 93 clinical psychologists were selected for graduate students at a public university in the city of Lima. They were evaluated with the Sánchez Cánovas Psycho-logical Well-being Scale (EBP) and the Wagnild and Young Resilience Scale (ER). The general results show a statistically significant moderate positive correlation between resilience and psychological well-being, that is, as, in psychologists working in the clinical setting, with postgraduate training, the capacity to be able to successfully transform the adversities that arise in life,
\end{abstract}

1 Psicóloga del Programa Nacional para la Promoción de oportunidades laborales del MTPE.
E-mail: ofeliapaolac.ps.p@gmail.com ORCID: https://orcid.org/0000-0002-9260-7840

(C) Los autores. Este artículo es publicado por la Revista de Investigación en Psicología de la Facultad de Psicología, Universidad Nacional Mayor de San Marcos. Este es un artículo de acceso abierto, distribuido bajo los términos de la licencia Creative Commons Atribucion - No Comercia_Compartir Igual 4.0 Internacional. (http://creativecommons.org/licenses/by-nc-sa/4.0/) que permite el uso no comercial, distribución y reproducción en cualquier medio, siempre que la obra original sea debidamente citada. 
allowing them to adapt and project themselves successfully to their context also increases their perception of their personal satisfaction.

Keywords: Resilience; Well-being; Clinical psychologists; Well-being in couple relationships and Equanimity.

En el Perú la demanda por estudiar la carrera de psicología se ha ido incrementado en los últimos años, al extremo que la carrera profesional de psicología en la actualidad figura en el ranking de las carreras más demandadas del sistema universitario peruano (Instituto Nacional de Estadística e Informática, INEI, 2015, Moreno, 2015). Pero a pesar que la profesión presenta tendencia a un gran crecimiento, su posicionamiento aún no está consolidado. Existen más de 30 mil psicólogos colegiados en el país, pero la población peruana supera los 32 millones de habitantes. Al respecto el decano de la Facultad de Psicología de la universidad Nacional Mayor de San Marcos, Alberto Quintana (Andina, 2019), en entrevista grabada, mencionaba que dadas las normas vigentes que mandaban la presencia de psicólogos por cada colegio, centro de salud, corte de justicia, penales, clubes deportivos, etcétera y el déficit de psicólogos para cubrir esa demanda, profesionales de otras áreas tratan de suplir ese déficit, pero en la mayoría de la veces no lo hacen de la manera adecuada.

Más aun, las condiciones laborales precarias de los profesionales de la salud, no favorecen su bienestar. Según Priale (2019), en nuestro país, los bajos ingresos económicos, siguen siendo uno de los principales motores de la emigración de los trabajadores de la salud; quienes, con el fin de compensar los insuficientes ingresos, optan con frecuencia por tener múltiples trabajos, situación que actúa en desmedro de su salud y del desempeño de sus actividades, con restricciones en la calidad del tiempo del que disponen.

Rionda, González, Granda, Fernández, y Díaz, (2019) y Otero, (2013), hallaron en el ejercicio de la psicoterapia, abatimiento emocional en aproximadamente cerca de la mitad de psicólogos que laboran en el área médico hospitalaria, razón por la cual, es menester analizar qué aspectos no atendidos del bienestar de estos profesionales pueden estar favoreciendo estos hechos. La psicología clínica es una carrera que encierra muchos aspectos de peligro; en principio, el tipo de pacientes a los que presta atención son habitualmente violentos, solicitantes, abatidos y con insuficiencias muy particulares; el experto de la salud ejecuta intervenciones regulares, enfrenta profundas dificultades y genera una vinculación inmediata y privada con cada uno de sus clientes (Breéis y cols., 2009). De ahí, que resulta evidente la necesidad de otorgarle importancia al estudio del bienestar psicológico en los profesionales de la salud. Entendiendo por bienestar psicológico a la percepción subjetiva de encontrarse disfrutando de una vida feliz (Sánchez Cánovas, 1998). 
La situación de tensión Laboral de los psicólogos que laboran en el ámbito clínico que hemos reseñado, infiriendo a partir de las reflexiones de Wagnild y Young (1993), podría ser superada con la resiliencia, en esencia, la capacidad de lograr modificar con éxito los infortunios y sufrimientos propios e inevitables que se presentan en la vida, mostrando la resistencia, potencialidades y riquezas que les permitirían confrontar la fatalidad, sobreponerse, quedar fortalecidos y mirar con certidumbre el porvenir. En este contexto resulta importante hacerse la siguiente pregunta o problema de investigación: ¿Habrá asociación entre la resiliencia y el bienestar en psicólogos que laboran en el ámbito clínico?

Con respecto a la resiliencia el trabajo se sustenta en la teoría de Wagnild y Young (1993) que al igual que otros estudiosos consideran la Resiliencia como una característica de la personalidad, que permite una pronta recuperación, vuelta a la labor normal, así como de una adaptación exitosa a pesar de las adversas circunstancias ambientales, permitiéndole proyectarse exitosamente, la misma que implica múltiples dimensiones e involucra la interacción de aspectos personales así como contextuales, los cuales permiten a las personas afrontar adecuadamente eventos adversos. (Gonzáles-Arratia, 2016; Ortega y Mijares, 2018; Saavedra 2012; Asociación Americana de Psicología, 2009; Connor y Davidson, 2003 y Quiñonez, 2006).

Enfatizando en los aspectos personales, Wagnild y Young (1993) definen a la resiliencia como la habilidad de poder cambiar con éxito las adversidades que se presentan en la vida. Para estos estudiosos la resiliencia sería "una característica de personalidad que modera el efecto negativo del estrés y fomenta la adaptación" (p. 165).

Los cinco componentes presentes en la resiliencia (Figura 1) son:

Confianza en sí mismo: Creer en las propias capacidades, dependiendo de sí mismo reconociendo sus fuerzas y limitaciones.

Ecuanimidad: Sopesar la propia vida y experiencias, mantenerse tranquilo, moderando respuestas extremas ante la adversidad.

Perseverancia: Persistir luchando para reconstruir la vida de sí mismo, permanecer involucrado y practicar autodisciplina a pesar de la adversidad o desaliento.

Satisfacción personal: Comprender que la vida tiene un significado y evaluar las propias contribuciones, darse una razón para vivir.

Sentirse bien solo: Comprender que mientras se comunican ciertas experiencias en la vida, restan otras que deben afrontarse a solas; y que eso los hace libres y únicos. 


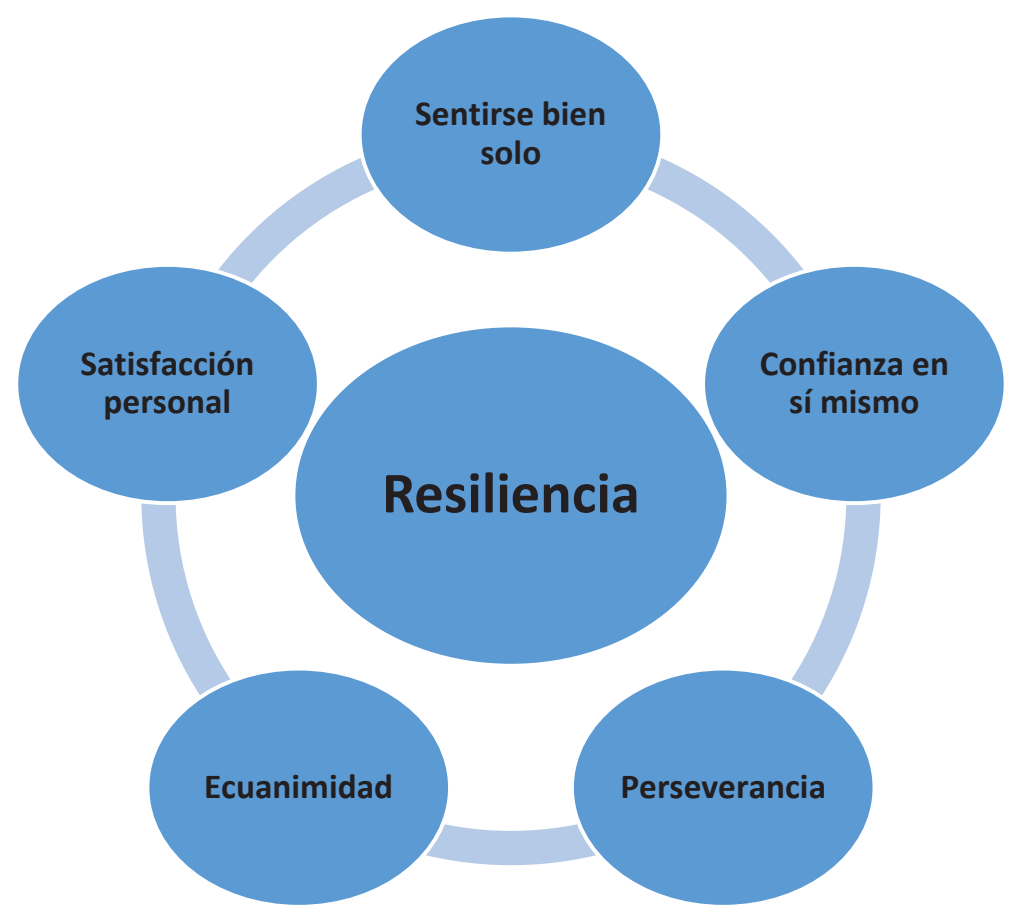

Figura 1. Componentes de la Resiliencia (Wagnild y Young, 1993)

En relación al Bienestar Psicológico el estudio se sostiene en la conceptualización de Meza (2012), quien como otros estudiosos sostiene que el bienestar psicológico se refiere a la apreciación que hacen las personas sobre sus vidas, la cual depende de las valoraciones cognitivas y por una estimación de las emociones y los estados de ánimo (Muñoz, 2017, García-Viniegras y González 2000, Gaxiola y Palomar, 2016). Concretizando lo anterior, Sánchez-Cánovas (1998) presenta una definición operacional del constructo Bienestar Psicológico que integra cuatro grandes componentes (Figura 2) tales como: Bienestar Subjetivo, Bienestar Material, Bienestar Laboral y Bienestar en las Relaciones de Pareja.

Los cuatro componentes presentes en el bienestar psicológico (Figura 2) son:

Bienestar subjetivo: la satisfacción con la percepción de su estado psicológico, pensamientos y equilibrio emocional, en un momento dado de sus vidas.

Bienestar material: la satisfacción con la percepción de sus ingresos económicos, bienes y posesiones cuantificables.

Bienestar Laboral: la satisfacción con la percepción de su trabajo o sus experiencias laborales. 
Bienestar de pareja: la satisfacción con la percepción de sus relaciones de pareja, en un momento dado de sus vidas.

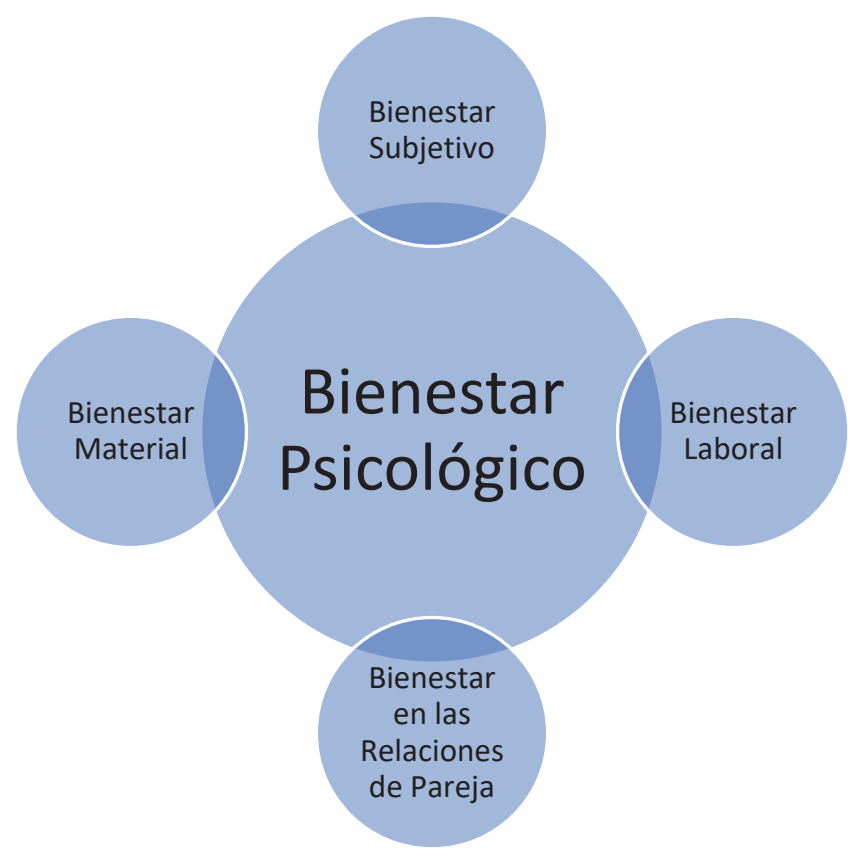

Figura 2. Componentes del bienestar psicológico (Sánchez, 1998).

Cohn, Fredrickson, Brown, Mikels \& Conway (2009), relacionan estos constructos, afirmando que la concordancia entre la resiliencia y las emociones positivas se reafirma del mismo modo en el tiempo formando espirales positivas ascendentes en las que la resiliencia y las emociones óptimas se influencian y se forman una a la otra. En consecuencia, sería lícito postular las siguientes hipótesis:

- A mayor resiliencia será mayor el bienestar psicológico en los sujetos de la muestra

- Se esperaría encontrar en especifico covariaciones positivas entre los componentes de la resiliencia: Satisfacción Personal, Sentirse Bien Solo, Confianza En Si Mismo, Perseverancia y Ecuanimidad y los componentes del Bienestar Psicológico: Bienestar Subjetivo, Bienestar Material, Bienestar Laboral y el Bienestar en Relaciones de Pareja.

Por lo expuesto líneas arriba, la investigación se realiza con el objetivo primordial de determinar la asociación entre la resiliencia y el bienestar psicológico en psicólogos que laboran en el área clínica. 


\section{MÉTODO}

\section{Diseño}

El estudio fue ex-post-facto mediante un diseño correlacional que permitió valorar el grado de relación entre las variables. Los estudios correlacionales, miden cada una de las variables y, después, cuantifican y analizan la relación entre ellas (Matheson, Bruce y Beauchamp, 1983 y Piscoya, 1987).

\section{Población}

La población considerada en el presente estudio, fue constituida por 135 psicólogos que laboran en el ámbito clínico, procedentes de Ministerio de Salud (MINSA), Seguro Social de Salud (EsSalud), clínicas privadas y consultorios particular. Procedentes de distintos programas de la unidad de posgrado del semestre 2018-1 de una universidad pública, a saber: Doctorandos en psicología, maestritas mención en psicología clínica, neurodesarollo, segunda especialidad en problemas de aprendizaje, psicología clínica, en patologías del lenguaje, etc.

\section{Muestra}

La muestra de estudio fueron 93 psicólogos que laboran en el ámbito clínico en distintos Centros de Salud de la Ciudad de Lima, que además eran alumnos de posgrado de una Universidad pública, 47 de los cuales fueron varones y 46 fueron mujeres, con edades comprendidas entre 25 a 70 años. Del total de alumnos de la unidad de posgrado estudiantes del doctorado, de la maestría en psicología clínica y de la salud, así como de la segunda especialidad en Psicología de la misma mención, y otros programas con mención cercana, fueron excluidos quienes no se encontraban en ese momento trabajando en algún centro de salud en la atención directa a pacientes, haciéndose el estudio con los 93 psicólogos restantes. Para este tipo de estudio se utilizó un muestreo no probalístico (Cochran, 1981).

\section{Instrumentos}

Escala de Bienestar Psicológico construida por Sánchez-Cánovas en el año de 1998, compuesta por 65 ítems, con estimaciones que comprenden desde nunca o casi nunca (1) a siempre (5). Valora las siguientes medidas: El Bienestar material, el Bienestar laboral, el Bienestar subjetivo y las Relaciones con la pareja.

La labor del análisis psicométrico realizada por Sánchez-Cánovas, (1998), le conllevo a sentar la validez del constructo empleando el método de criterio concurrente del constructo propuesto, para ello se formuló una investigación comparativa con los resultados de diferentes instrumentos que intentan considerar variables que hipotéticamente valoran constructos similares al que pretende evaluar la escala bienestar psicológico. Se tuvo en cuenta al Inventario de Felicidad de Oxford, a modo de una media del bienestar subjetivo. La correlación 
con el inventario de Felicidad de Oxford, es elevada y significativa $(\mathrm{r}=.90, \mathrm{p}<$ .001). También con otra medida afín con el bienestar psicológico, satisfacción con la vida (Lucas, Diener,. y Suh, 1996), la correlación estuvo más moderada, no obstante, significativa $(\mathrm{r}=.451, \mathrm{p}<.001)$. Se concluyó que, si bien moderadamente, todas las escalas se relacionan en el sentido deseado con el afecto tanto positivo como negativo. Respecto a la confiabilidad para cada subescala del EBP se encontraron coeficientes altos de Alfa de Cronbach, a saber: Bienestar material (.928), Bienestar subjetivo (.923), Bienestar laboral (.906) y Relaciones con la pareja (.993).

Escala de Resiliencia construida por Wagnild y Young (1988), examinada por los propios autores en el año de 1993. Consta de 25 ítems, que registran, en una escala tipo Likert de 7 puntos, en el cual 1 es en desacuerdo, y un puntaje máximo de acuerdo de 7. Los evaluados revelarán el grado de asentimiento con el ítem, puesto que todos los ítems son catalogados positivamente; los más elevados puntajes serán señales de una mayor Resiliencia. En cuanto el rango de puntaje cambia entre 25 y 175 puntos. El instrumento de Resiliencia posee como componentes: la Confianza en sí mismo, la Satisfacción personal, el Sentirse bien solo, la Perseverancia y la Ecuanimidad.

En primera instancia se estableció la validez del contenido, ya que los ítems fueron escogidos en concordancia con aquellos que mostraban las conceptualizaciones de Resiliencia, a criterio de los investigadores, dos psicometristas y dos enfermeras. Los ítems de la escala ER poseen un estilo de redacción positiva. La validez concurrente se expresa por los elevados índices de correlación de la Escala Resiliencia (ER) con medidas apropiadamente establecidas de constructos afines con la Resiliencia, es decir, correlacionando con medidas conveniente determinadas de constructos emparentados con la Resiliencia, tales como: La satisfacción, $\mathrm{r}=0.59$; la autoestima $\mathrm{r}=0.57$; la moral, $\mathrm{r}=0.54$; la salud $\mathrm{r}=0.50$; la percepción al estrés $\mathrm{r}=0.67$ y la Depresión $\mathrm{r}=0.36$. Para otra evaluación de la validez de la escala ER, se empleó el análisis factorial para estimar la disposición interna del instrumento, con el criterio de componentes principales y rotación oblimin, evidenciando 5 factores que no se presentan correlacionados entre sí.

Novella, (2002), refiere que en la investigación piloto se logró una confiabilidad por el criterio de la consistencia interna con un coeficiente alfa de Cronbach de 0.89. Los análisis referidos por Wagnild y Young proporcionaron confiabilidades de 0.86 y 0.85 en dos muestras de estudiantes femeninas profesionales; 0.85 en cuidadores con diagnóstico de Alzheimer; 0.90 en madres principiantes post parto; y con un indicador de 0.76 en moradores de alojamientos estatales. Conjuntamente, con el procedimiento test-retest la confiabilidad fue valorada por los responsables en una investigación longitudinal en mujeres en estado de gestación antes y después del parto, logrando correlaciones de puntajes que oscilan de 0.67 a 0.84 , en consecuencia, son fuertes. 
En la UNMSM, en la facultad de Psicología, se ejecuta en el 2004 la adaptación de la Escala de Resiliencia con estudiantes de secundaria de ambos sexos, entre 15 y 18 años de edad, obteniéndose un coeficiente alfa de Cronbach de 0.83 .

En este estudio se volvió a estimar la confiabilidad de los instrumentos empleando el Alfa de Cronbach, obteniendo resultados similares en ambas escalas, siendo la confiabilidad de .960 para la Escala de Bienestar Psicológico de Sánchez-Cánovas de 1998 y de ,944 para la Escala de Resiliencia de Wagnild y Young de 1988, de lo cual se deriva que los instrumentos tienen una confiabilidad bastante fuerte. En cuanto a la validez de ambas escalas, se recorrió a la validez de contenido por criterio de jueces, encontrándose un índice de coeficiente $\mathrm{V}$ de Aiken de 0.97 para la Escala de Bienestar Psicológico y de 0.95 para la Escala de Resiliencia.

\section{Procesamiento y análisis de datos}

Los resultados de la presente investigación fueron analizados mediante el programa estadístico SPSS v.24. En primer lugar, se probó la distribución normal de las variables con la prueba de kolmogorov-Smirnov, encontrando que no se rechazaron las hipótesis nulas, por lo cual las pruebas de correlación de las variables se realizaron con el coeficiente de correlación no paramétrico de Spearman, finalmente para la representación sumaria de los datos se utilizaron tablas.

\section{RESULTADOS}

\section{Descriptivos}

En la Tabla 1, los resultados obtenidos al evaluar la variable de resiliencia en la muestra total de psicólogos que realizan su ejercicio profesional en el ámbito clínico y son además estudiantes de la unidad de posgrado de una universidad pública, se encuentra que los mismos presentan una tendencia hacia los niveles bajo y medio de resiliencia. Mostrando así mismo, la predominancia de puntajes bajos o muy bajos en las dimensiones de satisfacción personal 39.0\% y sentirse bien solo $54.3 \%$, mientras que, por el contrario, se observan puntajes en niveles altos y muy altos de resiliencia en las dimensiones confianza en sí mismo 44,0 \%, perseverancia $44,1 \%$ y ecuanimidad $35.8 \%$. Se concluye, que aproximadamente un poco más de la mitad de la muestra evaluada evidencia niveles bajos y muy bajos de resiliencia.

De otro lado, en referencia a la variable Bienestar Psicológico en general, en la muestra (Tabla 2), se evidencia una disposición hacia los grados altos de bienestar, con $50.8 \%$ de los sujetos presentando puntajes altos y muy altos. Y así mismo, se encontró que en las dimensiones Bienestar subjetivo, bienestar laboral y bienestar en relaciones de pareja se ubican en los grados alto y muy alto de bienestar, con el 
$44 \%$, el $44 \%$ y el $42.3 \%$, respectivamente. Se concluye, que aproximadamente más de la mitad ostenta tendencia a niveles altos y muy altos de bienestar psicológico.

\section{Tabla 1}

Niveles de la Resiliencia conforme a la muestra total

\begin{tabular}{lcccccc}
\hline & \multicolumn{5}{c}{ Niveles de Resiliencia } \\
& Muy bajo \% & Bajo \% & Medio \% & Alto \% & Muy alto \% & Total \% \\
\cline { 2 - 7 } Resiliencia & 15,3 & 84,7 & 0 & 0 & 0 & 100 \\
Satisfacción Personal & 13,6 & 25,4 & 30,5 & 28,8 & 1,7 & 100 \\
Sentirse Bien Solo & 13,6 & 40,7 & 35,6 & 10,2 & 0 & 100 \\
Confianza En Sí Mismo & 18,6 & 18,6 & 18,6 & 20,3 & 23,7 & 100 \\
Perseverancia & 13,6 & 23,7 & 18,6 & 27,1 & 16,9 & 100 \\
Ecuanimidad & 11,9 & 20,3 & 32,2 & 18,6 & 16,9 & 100 \\
\hline
\end{tabular}

Tabla 2

Niveles de Bienestar Psicológico según muestra total

\section{Grados}

\begin{tabular}{lcccccc} 
& Muy bajo \% & Bajo \% & Medio \% & Alto \% & Muy alto \% & Total \% \\
\cline { 2 - 7 } Bienestar Psicológico & 8,5 & 11,9 & 28,8 & 23,7 & 27,1 & 100 \\
Bienestar Subjetivo & 16,9 & 23,7 & 15,3 & 23,7 & 20,3 & 100 \\
Bienestar Material & 16,9 & 22 & 20,3 & 20,3 & 20,3 & 100 \\
Bienestar Laboral & 13,6 & 16,9 & 25,4 & 18,6 & 25,4 & 100 \\
Bienestar en Relaciones de Pareja & 27,1 & 5,1 & 25,4 & 22 & 20,3 & 100 \\
\hline
\end{tabular}

En función a los resultados encontrados, empleando el coeficiente de correlación de Spearman con un nivel de significancia de ,001, se puede afirmar una correlación positiva, moderada $(, 40)$, estadísticamente significativa, entre el bienestar psicológico y la resiliencia.

Más aún, en la Tabla 3, que muestra las correlaciones entre los sub-test de ambas variables, se aprecian correlaciones positivas en su mayoría moderadas o altamente significativas (coeficientes de correlación que van de .304 a .586), con niveles de significancia de .000 ó .001 y ajustes adecuados a juzgar por los tamaños del efecto estimados, entre todos los componentes del bienestar Psicológico y todos los componentes de la resiliencia, a excepción de la falta de correlación, entre la dimensión Bienestar en las relaciones de pareja y la resiliencia en general ni ninguno de sus sub-test, tampoco, entre la dimensión ecuanimidad de la resiliencia y el Bienestar psicológico en general ni ninguno de sus sub-test. 
Tabla 3

Correlaciones entre los sub-test de sub-test de Bienestar Psicológico y los sub-test de resiliencia

\begin{tabular}{|c|c|c|c|c|c|c|c|}
\hline \multicolumn{8}{|c|}{ Correlaciones } \\
\hline & & & $\begin{array}{c}\text { Satisfacción } \\
\text { Personal }\end{array}$ & Ecuanimidad & $\begin{array}{c}\text { Sentirse } \\
\text { Bien Solo }\end{array}$ & $\begin{array}{l}\text { Confianza En } \\
\text { Sí Mismo }\end{array}$ & Perseverancia \\
\hline \multirow[t]{16}{*}{$\begin{array}{l}\text { Rho de } \\
\text { Spearman }\end{array}$} & \multirow[t]{4}{*}{$\begin{array}{l}\text { Bienestar } \\
\text { Subjetivo }\end{array}$} & $\begin{array}{l}\text { Coeficiente de } \\
\text { correlación }\end{array}$ &, $485^{* *}$ & ,140 &, $586^{* *}$ &, $607^{* *}$ &, $533^{* *}$ \\
\hline & & Sig. (unilateral) &, 000 & ,145 &, 000 &, 000 & ,000 \\
\hline & & R cuadrado & 0,24 & 0,02 & 0,34 & 0,37 & 0,28 \\
\hline & & $\mathrm{N}$ & 93 & 93 & 93 & 93 & 93 \\
\hline & \multirow[t]{4}{*}{$\begin{array}{l}\text { Bienestar } \\
\text { Material }\end{array}$} & $\begin{array}{l}\text { Coeficiente de } \\
\text { correlación }\end{array}$ &, $421^{* * *}$ & ,120 &, $438^{* * *}$ &, $507^{* *}$ &, $304^{* *}$ \\
\hline & & Sig. (unilateral) &, 000 & ,183 &, 000 &, 000 &, 010 \\
\hline & & R cuadrado & 0,18 & 0,01 & 0,19 & 0,26 & 0,09 \\
\hline & & $\mathrm{N}$ & 93 & 93 & 93 & 93 & 93 \\
\hline & \multirow[t]{4}{*}{$\begin{array}{l}\text { Bienestar } \\
\text { Laboral }\end{array}$} & $\begin{array}{l}\text { Coeficiente de } \\
\text { correlación }\end{array}$ &, $357^{* *}$ &, 017 &, $266^{*}$ &, $574^{* *}$ &, $473^{* *}$ \\
\hline & & Sig. (unilateral) & ,003 & ,448 & ,021 &, 000 &, 000 \\
\hline & & R cuadrado & 0,13 & 0,00 & 0,07 & 0,33 & 0,22 \\
\hline & & $\mathrm{N}$ & 93 & 93 & 93 & 93 & 93 \\
\hline & \multirow{4}{*}{$\begin{array}{l}\text { Bienestar en } \\
\text { las Relaciones } \\
\text { de Pareja }\end{array}$} & $\begin{array}{l}\text { Coeficiente de } \\
\text { correlación }\end{array}$ &,- 044 &,- 168 &,- 052 &, 179 & ,118 \\
\hline & & Sig. (unilateral) & ,369 & ,101 & ,349 &, 087 & ,188 \\
\hline & & R cuadrado & 0,00 & 0,03 & 0,00 & 0,03 & 0,01 \\
\hline & & $\mathrm{N}$ & 93 & 93 & 93 & 93 & 93 \\
\hline
\end{tabular}

**. La correlación es significativa en el nivel 0,01 (1 cola).

*. La correlación es significativa en el nivel 0,05 (1 cola).

\section{DISCUSIÓN}

El objetivo principal en esta investigación ha sido establecer si existe relación entre el bienestar psicológico y la resiliencia en psicólogos del ámbito clínico de Lima Metropolitana Los resultados demuestran que en los psicólogos clínicos con formación de posgrado a medida que aumenta su satisfacción con la Percepción que tienen de sí mismos aumenta también su capacidad de poder transformar con éxito las calamidades que se les presentan en el trascurso de la vida, que les permite adaptarse y proyectarse exitosamente, vale decir, que existe en ellos una correlación positiva moderada entre el bienestar psicológico y la resiliencia. Los hallazgos coinciden con los de Cohn, Fredrickson, Brown, Mikels \& Conway (2009), cuando sostienen que la reciprocidad entre las emociones positivas y la resiliencia se fortalece a lo largo del tiempo propiciando espirales óptimas ascendentes en las que las emociones positivas y la resiliencia se influencian y se producen una a la 
otra, concurriendo que la resiliencia sería una particularidad de la personalidad coligada a un nivel alto de Bienestar percibido.

Los resultados de la investigación además indican correlaciones altamente significativas mayormente fuertes y ajuste adecuado a juzgar por el tamaño del efecto estimado, entre el bienestar subjetivo y la satisfacción personal, sentirse bien solo, confianza en sí mismo y perseverancia. Vale decir, que mientras mayor sea la satisfacción con la percepción de su estado psicológico, pensamientos y equilibrio emocional, en un momento dado de sus vidas, mayor será su tendencia a: a) entender que la vida posee un significado, apreciar las personales contribuciones y darse una razón para vivir, b) comprender que mientras se comparten determinadas experiencias en la vida, restan demás que deben revolverse a solas y que eso hace a las personas libres y únicas, c) Creer en las propias habilidades, dependiendo de su persona, identificando sus fortalezas y barreras, y d) persistir en la luchar con el objetivo de rehacer la vida de sí mismo, persistir implicado y realizar autodisciplina pese al desaliento y la adversidad. Esta asunción concuerda con las conclusiones de Cohn y colaboradores (2009), quienes en su investigación encontraron que las emociones positivas generan aumentos en el nivel de Resiliencia y en el nivel de la satisfacción con la vida, y, así mismo, con los de Liossis, Shochet, Millear y Biggs (2009), quienes demostraron que desarrollando la resiliencia en personas adultas se originan resultados positivos congruentes con el bienestar de la persona, en el plano personal y del trabajo.

Así mismo, indican correlaciones altamente significativas mayormente moderadas y ajuste adecuado a juzgar por el tamaño del efecto estimado (a excepción de con perseverancia), entre el bienestar material y la satisfacción personal, sentirse bien solo, confianza en sí mismo y perseverancia. Vale decir, que mientras mayor sea la satisfacción con la percepción de sus ingresos económicos, bienes y posesiones cuantificables., mayor será su tendencia a: a) entender que la vida posee un significado, apreciar las propias contribuciones y darse una razón para vivir, b) comprender que mientras se comunican determinadas experiencias en la vida, restan algunas otras que deben revolverse a solas y que eso hace a las personas libres y únicas, c) Creer en las propias habilidades, dependiendo de sí mismo identificando sus potencialidades e impedimentos, y d) persistir en la lucha para rehacer la vida de sí mismo, insistir implicado y ejercer autodisciplina a pesar del desaliento o la adversidad. Coincidiendo con los hallazgos de Murillo y Molero (2012) quienes en inmigrantes colombianos residentes en Ecuador encontraron que a altos niveles de satisfacción con la vida se correspondía un mayor bienestar material percibido.

De igual manera, indican correlaciones altamente significativas mayormente moderadas y ajuste adecuado a juzgar por el tamaño del efecto estimado (a excepción de con sentirse bien solo), entre el bienestar laboral y la satisfacción personal, sentirse bien solo, confianza en sí mismo y perseverancia. Vale 
decir, que mientras mayor sea la satisfacción con la percepción de su trabajo o sus experiencias laborales, mayor será su tendencia a: a) advertir que la vida asume un significado, valorar las propias contribuciones y darse una razón para vivir, b) comprender que mientras se comunican algunas experiencias en la vida, existen otras que deben enfrentarse a solas y que eso hace a las personas libres y únicas, c) Creer en las propias habilidades, dependiendo de sí mismo identificando sus fortalezas y limitaciones, y d) persistir en la luchar para enmendar la vida de sí mismo, perseverar involucrado y ejercer autodisciplina a pesar de la adversidad o el desaliento, coincidiendo con los hallazgos de Yousset y Luthans (2007) quienes demostraron que la resiliencia de los trabajadores adquiere una impresión positiva en la satisfacción, el compromiso y el bienestar en el ámbito laboral.

Mención aparte amerita la comprobación de que no existe relación alguna entre el bienestar en las relaciones de pareja y la satisfacción personal, sentirse bien solo, confianza en sí mismo, ecuanimidad y perseverancia, vale decir, no existe relación alguna entre la satisfacción con la percepción de sus relaciones de pareja, en un momento dado de sus vidas y a) entender que la vida posee un significado, apreciar las propias contribuciones y darse una razón para vivir, b) comprender que mientras se comunican ciertas experiencias en la vida, restan muchas otras que deben afrontarse a solas y que eso hace a las personas libres y únicas, c) Creer en las propias habilidades, dependiendo de sí mismo identificando sus potencialidades y barreras, d) Sopesar la propia vida y experiencias, mantenerse tranquilo, moderando respuestas extremas ante la adversidad, ni d) persistir en la lucha para rehacer la vida de sí mismo, persistir implicado y ejercer autodisciplina a pesar de la adversidad o el desaliento, Así como tampoco, existe relación alguna entre la habilidad de sopesar la propia vida y experiencias, mantenerse tranquilo, moderando respuestas extremas ante la adversidad y la satisfacción con la percepción de: a) su estado psicológico, pensamientos y equilibrio emocional, en un momento dado de sus vidas. b) sus ingresos económicos, bienes y posesiones cuantificables y c) su trabajo o sus experiencias laborales.

Una de las limitaciones de la presenta investigación estaría asociada a la posible deseabilidad social que pudo llevar a más de un profesional de la salud mental a responder de manera positiva a los instrumentos psicológicos empleados, lo cual podría haber mediado en parte en algunos resultados.

\section{CONCLUSIONES}

- Queda pendiente comprobar si la relación demostrada entre el bienestar y la resiliencia en psicólogos del ámbito clínico se cumplen en grupos de diversas disciplinas. 
- Otra arista de investigación pendiente es comprobar si la ausencia de relación entre la resiliencia de los profesionales de la salud y su bienestar en la relación de pareja se explica por una mayor subjetividad de la satisfacción en las relaciones de pareja que en los otros componentes del bienestar.

- Una última arista de investigación pendiente es comprobar si la ausencia de relación entre la ecuanimidad de los profesionales de la salud y su bienestar Psicológico, Subjetivo, Material, Laboral y en Relaciones de Pareja, podría estar asociada, entre otras posibles explicaciones al hecho que siendo los sujetos de la muestra psicólogos clínicos, de los que se espera ayuden a sus clientes a mantenerse tranquilos, moderando sus respuestas extremas ante la adversidad, por deseabilidad social o sensibilidad a sus reacciones emocionales no hayan sido del todo objetivos al responder a los ítems de la sub-escala de ecuanimidad.

\section{Agradecimientos / Acknowledgments:}

La autora agradece a todos los profesionales psicólogos clínicos por la participación en responder las escalas psicológicas, utilizadas en la investigación.

\section{Fuentes de financiamiento / Funding:}

El presente estudio y artículo han sido financiados por la misma autora.

\section{Rol de los autores / Authors Roles:}

La autora realizó, la aplicación de las pruebas, redacción, análisis y procesamiento de data, examen estadístico, análisis e interpretación de los resultados, corrección y elaboración de artículo.

\section{Aspectos éticos / legales; Ethics / legals:}

Se ha considerado y respetado lo estipulado por las normativas éticas que reglamentan el ejercicio profesional acorde al Código de Ética del Colegio de Psicólogos del Perú. Asegurando el anonimato de los involucrados e instituciones implicadas en la muestra, haciendo referencia al amparo de la confidencialidad de la información personal y también de orden institucional.

\section{Conflicto de intereses / Competing interests:}

La autora declara bajo juramento no haber incidido en conflicto de interés al realizar la presente investigación.

\section{REFERENCIAS}

American Psychological Association (2009). Resilience. Recuperado el 16/07/2013, Recuperado de https://www.apa.org/practice/programs/campaign/resilience\# 
Andina. (Productor). (2019). Andina. Todo lo que debes saber para estudiar Psicología. De https://www.facebook.com/agenciandina/videos/1519630548173980/?extid=8k1X7Aa71y Ua9Eqa

Breéis, C., Porcellana, M., Gambini, O., Meida, L., Muffatti, R., Peirone, A. Zanini, S., Erlicher, S., Scarone, S. y Altamira, C. (2009). Burnout among psychiatrists in Milan: A multicenter survey. Psychiatric Services, July 1, 60(7). DOI: 10.1176 / ps.2009.60.7.985

Cochran, W. (1981) Técnicas de muestreo. Compañía editorial Continental, S.A.

Cohn, M. A., Fredrickson, B. L., Brown, S. L., Mikels, J. A. y Conway, A. M. (2009). Happiness unpacked: Positive emotions increase life satisfaction by building resilience. Emotion, 3, 361-368. DOI: 10.1037 / a0015952

Connor, K. M. y Davidson, J. R. T. (2003). Development of a new resilience scale: The Connor-Davidson Resilience Scale (CD-RISC). Depression and Anxiety, 18, 76-82. DOI: 10.1002 / da.10113

Flores, M. (2008). Resiliencia y proyecto de vida en estudiantes del tercer año de secundaria de la UGEL 03 (Tesis de Licenciatura). Universidad nacional Mayor de San Marcos, Lima, Perú. Recuperado de http://cybertesis.unmsm.edu.pe/bitstream/ cybertesis/621/1/Flores_cm.pdf

García-Viniegras, C., \& González, I. (2000). La categoría bienestar psicológico, su realidad con otras categorías sociales. Revista Cubana de Medicina General Integral, 16(6), 586-592. ISSN 1561-3038. Recuperado de http://scielo.sld.cu/scielo.php?script=sci arttext\&pid=S0864-21252000000600010

Gaxiola, J., y Palomar, J. (2016). El Bienestar Psicológico: Una mirada desde Latinoamérica. México: Editorial Qartuppi. DOI: 10.29410/QTP.16.09

Gonzales-Arratia, N. J. (2016). Resiliencia y Personalidad en niños y adolescentes. Cómo desarrollarse en tiempos de crisis (2da. Ed.). México D.F.: Ediciones y gráficos Eón S.A. de C.V. ISBN 978-607-9426-54-5

Hernández, R., Fernández, C. y Baptista, M. (2018) Metodología De La Investigación. 5to Edicion. McGraw-Hill

Instituto Nacional de Estadística e Informática (2015). Encuesta Nacional a Egresados Universitarios y Universidades, 2014. Recuperado de https://www.inei.gob.pe/media/MenuRecursivo/publicaciones_digitales/Est/Lib1298/Libro.pdf

Liossis, P.L., Shochet, I.M., Millear, P.M \& Biggs, H. (2009).The promoting Adult Resilience (PAR) Program: The effectiveness of the Second, Shorter Pilot of Workplace Prevention Program. Behaviour Change, 26(2), 97-112. doi.org/10.1375/ bech.26.2.97

Moreno, B. Z. (2015). La formación del psicólogo en el Perú. Integración Académica en Psicología., 3(9). Recuperado de https://integracion-academica.org/anteriores/2uncategorised/106-la-formacion-del-psicologo-en-el-peru 
Meza, B. (2012). Bienestar psicológico en practicantes de danza contemporánea (Tesis de pregrado). Pontificia Universidad Católica del Perú. Lima, Perú. Recuperado de http://hdl.handle.net/20.500.12404/1217

Muñoz, A. (2017, 14 de febrero). ¿Qué es el bienestar psicológico? El modelo de Carol Ryff. Mensaje dirigido a https://www.aboutespanol.com/que-es-el-bienestarpsicologico-el-modelo-de-carol-ryff-2396415

Murillo, J. y Molero, F. (2012) La satisfacción vital: su relación con el prejuicio, la identidad nacional, la autoestima y el bienestar material, en inmigrantes, Acta Colombiana de Psicología 15 (2): 99-108. ISSN electrónico: 1909-9711 Recuperado de http:// www.scielo.org.co/pdf/acp/v15n2/v15n2a10.pdf

Ortega, S., y Mijares, B. (2018). Concepto de resiliencia: desde la diferenciación de otros constructos, escuelas y enfoques. Revista Científica Electrónica de Ciencias Humanas, 39 (13), 30-43. ISSN 1856-1594 Recuperado de http://www.revistaorbis.org.ve/ pdf/39/art3.pdf

Otero, B. G. I., Grajeda, G. G., Canto, V. B., Venegas, J. M., \& Luque, C. M. (2013). Burnout en psicólogos de la salud: características laborales relacionadas. Psicología y salud, 23(2), 217-226. doi.org/10.25009/pys.v23i2.503

Piscoya, H. (1987) Investigación cientifica y educacional. Amaru Editores. Recuperado de https://issuu.com/unmsm-prolex/docs/metodolog_a_de_la_investigaci_n_c

Priale, J. (14 de agosto de 2019). Gobierno incrementará sueldos de profesionales del sector salud.Gestión, Recuperado de https:/gestion.pe/economia/gobierno-incrementara-sueldos-de-profesionales-del-sector-salud-noticia/

Quiñonez, M (2006). Creatividad y resiliencia. Análisis de 13 casos colombianos. Tesis doctoral. Universidad Autónoma de Madrid. Madrid, España. Recuperado de http:// hdl.handle.net/10486/2631

Roberto Hernández Sampieri y Mendoza (2018), Metodología de la Investigación Científica. Mc Graw Hill. ISBN: 978-1-4562-2396-0

Rionda, I. S., González, J. V. G., Granda, F. J. L., Fernández, E. A., \& Díaz, Á. L. (2019). Grado de burnout en especialistas en formación de medicina y psicología clínica. Educación Médica. doi.org/10.1016/j.edumed.2019.05.002

Saavedra, E. (2012). Una aproximación al estado del arte de la investigación en resiliencia. En J. Catalán. (Ed.). Investigación orientada al cambio en Psicología Educacional. La Serena: Universidad de la serena. ISBN: 9789567393725

Sánchez-Cánovas, José. (1998) Escala de Bienestar Psicológico. Madrid. TEA.

Wagnild, G.M. \& Young, H.M. (1993). Development and psychometric evaluation of the Resilience Scale. Journal of Nursing Measurement, 1 (2), 165-178. PMID: 7850498. Recuperado de https://sapibg.org/download/1054-wagnild_1993_resilience_scale_2.pdf

Youssef,C.M. \& Luthans, F.(2007).Positive Organizational Behavior in the Workplace: The Impact of Hope, Optimism, and Resilience. Journal of Management, 33(5), 774800. DOI: 10.1177 / 0149206307305562 
